\title{
Situated Messages for Asynchronous Human-Robot Interaction
}

\author{
Nicolai Marquardt, James Young, Ehud Sharlin, Saul Greenberg \\ Interactions Lab, University of Calgary \\ 2500 University Drive NW, Calgary, AB, T2N 1N4, Canada \\ \{nicolai.marquardt, james.young, ehud.sharlin, saul.greenberg\}@ucalgary.ca
}

\begin{abstract}
An ongoing issue in human robot interaction (HRI) is how people and robots communicate with one another. While there is considerable work in real-time human-robot communication, fairly little has been done in asynchronous realm. Our approach, which we call situated messages, lets humans and robots asynchronously exchange information by placing physical tokens - each representing a simple message - in meaningful physical locations of their shared environment. Using knowledge of the robot's routines, a person can place a message token at a location, where the location is typically relevant to redirecting the robot's behavior at that location. When the robot passes nearby that location, it detects the message and reacts accordingly. Similarly, robots can themselves place tokens at specific locations for people to read. Thus situated messages leverages embodied interaction, where token placement exploits the everyday practices and routines of both people and robots. We describe our working prototype, introduce application scenarios, explore message categories and usage patterns, and suggest future directions.
\end{abstract}

\section{Categories and Subject Descriptors \\ I.2.9 [Robotics]: Operator Interfaces}

\section{General Terms: Experimentation, Human Factors, Design}

Keywords: Human-Robot Interaction, Asynchronous Interaction, Situated Messages, RFID.

\section{INTRODUCTION}

While once the domain of science fiction, robots are now established in the domestic setting [5]. In particular, commercial service robots, such as the iRobot Roomba, often perform tasks in households [5], [9]. Because most of today's tasks are simple ones, such robots are predominantly autonomous. However, we anticipate that future robots will be able to perform a variety of different, more complex tasks to support families in their homes [5].

A consequence of this richer capability will be an increasing need for household robots and its human inhabitants to communicate and coordinate with one another. In particular, people need easy ways to issue task instructions to robots, and to get feedback from robots about their activities and/or problems that have occurred. This can be difficult to achieve, given that programming of robots is a complex and/or tedious task. For today's commercial household robots, interaction is reminiscent of computing in the 1950s: people communicate through small buttons, error code displays, and blinking lights on the robot's body. Other options

Copyright is held by the author/owner(s). HRI'09, March 11-13, 2009, La Jolla, California, USA. ACM 978-1-60558-404-1/09/03. include controlling a robot directly through a computer GUI, which is a tedious and heavy-weight way to issue occasional commands to a robot. Furthermore, these methods are usually restricted to real-time human-robot communication, where issued commands are immediately executed at the current location of the robot [4]. Our particular concern - and the focus of this paper - is asynchronous interaction, where a person can issue one or more location-specific commands to a robot.

Our approach, which we call situated messages, lets humans and robots asynchronously exchange information by placing physical tokens - each representing a simple message - in meaningful physical locations of their shared environment. Using knowledge of the robot's routines, a person can place a message token at a location, where the location is typically relevant to redirecting the robot's behavior at that location. The next section explains how this concept supports asynchronous HRI, and how it improves human-robot awareness [1]. Section 3 applies situated messages to domestic robots, while Section 4 explains our prototype implementation. We close with a discussion and future work.

\section{SITUATED MESSAGES}

Leaving messages at meaningful places in the environment is an important form of communication between home inhabitants [7]: the interaction with others takes place at the same location, but a different time [4]. Examples are bills to be paid left on the kitchen table, notes on the fridge, sticky notes on the TV screen, messages by the phone, or reminders at the door entrance. Message location is key, e.g., to identify its importance, meaning, context, and/or the recipient [3]. People also exploit their knowledge of other's routines as they pass by the location so they are read at opportune moments [3]. Such messages are examples of embodied interaction: "the way that physical and social phenomena unfold in real time and real space as a part of the world in which we are situated, right alongside and around us" [1].

Situated messages applies this concept to asynchronous locationbased HRI. People leave messages for robots at specific locations, where robots detect these messages and react accordingly. This human-robot communication method differs from existing training and programming approaches [8] in various aspects. First, the communication is asynchronous (different time) but collocated (in the same general environment) [4], [10]. Second, the physical location of messages in the environment is a crucial part of the message itself, telling the robot which physical location areas the message pertains to. Third, the physical presence of the situated messages themselves is important to the person, as it provides awareness [2] of the robot's previous activities and future actions. While this includes the messages left for the robot (in that they reflect the future activities of the robot), they can also be messages that robots leave for humans. 


\section{DOMESTIC ROBOTS}

We now focus on domestic robots [5], where we briefly categorize several human-to-robot situated messages as follows.

- Instructions: tasks ("clean this area carefully"), exceptions ("do not enter this room"), navigation ("return to home base")

- Context information: environment ("stairs"), warnings ("hot"), location ("living room")

- Training (e.g., "do this activity once a week")

- Conditions (e.g., "do this activity only if no one is at home")

Each of these messages can have additional attributes for timing and duration. Examples include instructions for permanent training vs. one-time commands. These categories are starting points: we anticipate additional message categories.

Robots can also leave messages in the environment for humans a form of interaction that in earlier work is described as robot thought crumbs [11]. We extend this research by giving the messages a physical representation besides the virtual message content. Such robot-to-human messages include:

- Status: completed tasks, explanations for incomplete tasks ("hard-to-clean area", "obstructions")

- Observations: important observed events ("accidentally broken vase", "defect power outlet")

- Requests: request for support by humans ("replace filter", "move furniture")

- Traces: leaving traces of robot's previous activities ("I was here", "repaired this on Tuesday")

\section{IMPLEMENTATION}

Our implemented platform is controlled by a small mobile computer attached to an iRobot Create. The prototype, illustrated in Figure 1, implements three important aspects to work with situated messages: reading messages, finding messages, and leaving messages in the environment.

We use Radio-Frequency Identification (RFID) technology, where RFID tags contain the messages for or from the robot. The robot reads tag information associated with an RFID message as it passes by it. An RFID reader, connected to the mobile computer, is attached to the front of the robot. When the robot discovers an RFID tag, the mobile computer interprets the message and transforms it into a command. Because the detection range for RFID is limited to only a few inches, we supplement RFID tags with a small infrared (IR) light, which allows the robot to find the tag from a further distance (or, find the tag within a larger area). To do this, we attached a Nintendo Wiimote to our robot, where we use the Wiimote's high-speed IR tracking camera to discover the IR tokens from a distance of up to two meters.

Our robot can also leave messages in the environment. To do this, we added a mechanical unit to the robot, consisting of a second RFID reader, two servo motors, and a magazine for a stack of RFID tokens. The robot can drop these tokens at locations to provide the various messages as mentioned in Section 3, which can be digitally read by people. This use of RFID is quite different to other approaches in robotics [6], where RFID tags were statically embedded in the environment or attached to objects, and did not represent messages for asynchronous HRI.

\section{DISCUSSION AND FUTURE WORK}

We argue situated messages can facilitate asynchronous communication between humans and robots. This physical

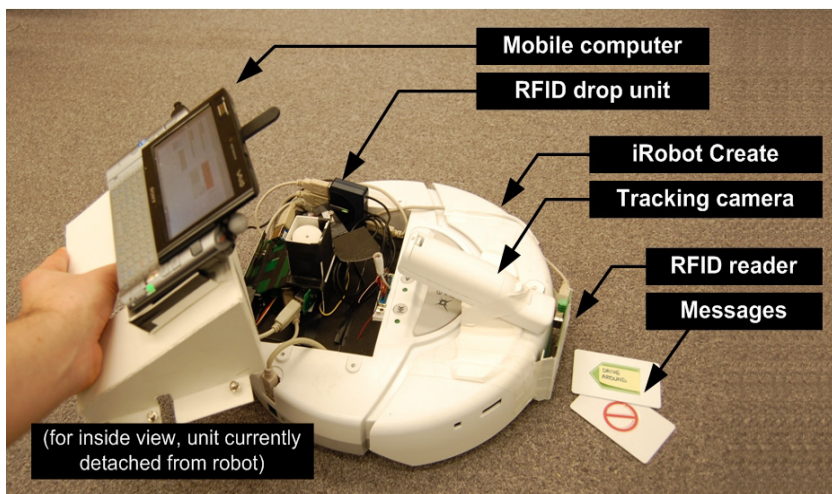

Figure 1. Robot prototype that implements concept of situated messages.

presence of the messages is important to provide human-robot awareness of past or future activities, where messages are embodied in the everyday routines and practices of both people and robots. Our prototype platform, albeit crude, illustrates that we can implement such a system using existing technologies.

Our future investigations will begin by critically evaluating our design implementation. Inspired by related HCI research [3], we are also interested in more explicitly investigating the possible occurrence of contextual locations for human-robot interaction. That is, we will explore how meaningful locations for robots can be exploited. Examples include room entrances, the robot home base, and the locations where robots perform their primary work.

Situated messages for HRI is not limited to domestic applications. For example, in search and rescue cases a direct communication network may not be available or may fail. Situated messages then might provide an alternate way for communication between members of the human-robot teams. Robots that searched at various locations can place messages when leaving a room or area, which could include information such as robot observations, warnings, etc. Other team members (humans, but also other robots) can immediately see these messages, read the digital content, and react accordingly.

\section{REFERENCES}

[1] Dourish, P. (2001) Where The Action Is: The Foundations of Embodied Interaction. MIT Press.

[2] Drury, J. L., Scholtz, J., and Yanco, H. A. (2003) Awareness in Human-Robot Interactions. Proc. of SMC '03.

[3] Elliot, K., Neustaedter, C. and Greenberg, S. (2005) Time, Ownership and Awareness: The Value of Contextual Locations in the Home. Proc. of UbiComp 2007. Springer.

[4] Ellis, C. A., Gibbs, S. J., and Rein, G. (1991) Groupware: some issues and experiences. Comm. ACM, 34, 1 .

[5] Forlizzi, J. and DiSalvo, C. (2006) Service robots in the domestic environment: a study of the roomba vacuum in the home. In Proc. of HRI '06. ACM.

[6] Koch, J., Wettach, J., Bloch, E., and Berns, K. (2007) Indoor Localisation of Humans, Objects, and mobile Robots with RFID Infrastructure. Proc. of HIS'07. IEEE.

[7] Perry, M. and Rachovides, D. (2007) Entertaining situated messaging at home CSCW: Journal of Collab. Comp., 16(1-2).

[8] Saunders, J., Nehaniv, C. L., and Dautenhahn, K. (2006) Teaching robots by moulding behavior and scaffolding the environment. Proc. of HRI '06. ACM.

[9] Sung, J., Grinter, R. E., Christensen, H. I., and Guo, L. (2008) Housewives or technophiles?: understanding domestic robot owners. Proc. HRI '08. ACM.

[10] Yanco, H.A., Drury, J. (2004) Classifying Human-Robot Interaction: An Updated Taxonomy. IEEE Conference on SMC.

[11] Young, J. E. and Sharlin, E. (2006) Sharing Spaces with Robots: an Integrated Environment for Human- Robot Interaction. Proc. of ISIE '06. MSR Press. 


\section{Situated Messages for}

Asynchronous Human-Robot Interaction

\section{Vision for Asynchronous Human-Robot Interaction}

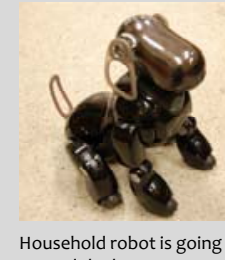

around the house...

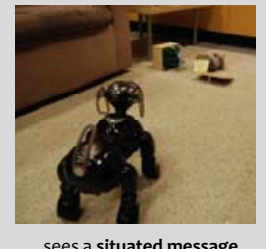

... sees a situated message left by the family.

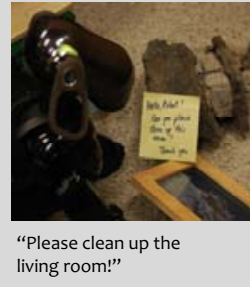

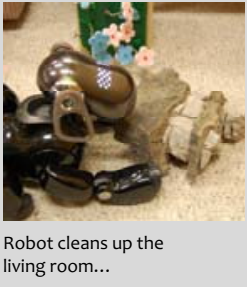
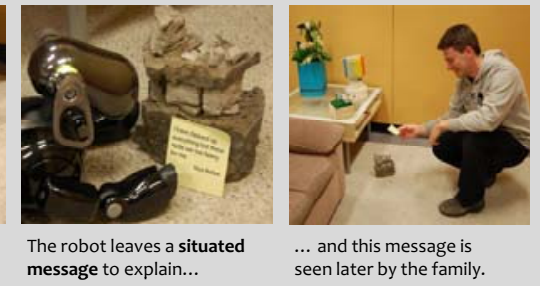

\section{Concept of Situated Messages}

- Humans and robots can exchange information asynchronously

- Communication at the same place, but at a different time

- People place physical tokens at meaningful locations in the shared environment - the physical location of message is crucial part of the message itself

- Situated messages leverage embodied interaction, as the message token placement exploits robot's and human's everyday routines

- Robots detect messages, interpret the message content and react accordingly

- Robots are able to leave "thought crumb" messages in the environment, increasing the human-robot awareness

\section{Open Questions}

What are meaningful locations of messages for robots? Homebase? Doors?

Role of the physical embodiment: physical vs. virtual messages.

Critical vs. non-critical tasks: how can situated messages complement direct humanrobot interaction?
Human-to-Robot Messages

instructions: tasks ("clean this area carefully"), exceptions ("do not enter rom"), navigation ("return to hom base")

formation: environment context information: "shot"), location ("living room") Training ("do this activity once a week") ditions ("do this activity conditions ("do this active")

\author{
Robot-to-Human Messages \\ incomplete tasks ("hsks, explanations for \\ "obstructions") ("hard-to-clean area", \\ Observations: important observed events \\ outlet") \\ Requests: request for support by human \\ ("replace filter", "move furniture") \\ Traces: leaving traces of robot's previous \\ activities ("I was here" "robot's previous \\ Tuesday")
}

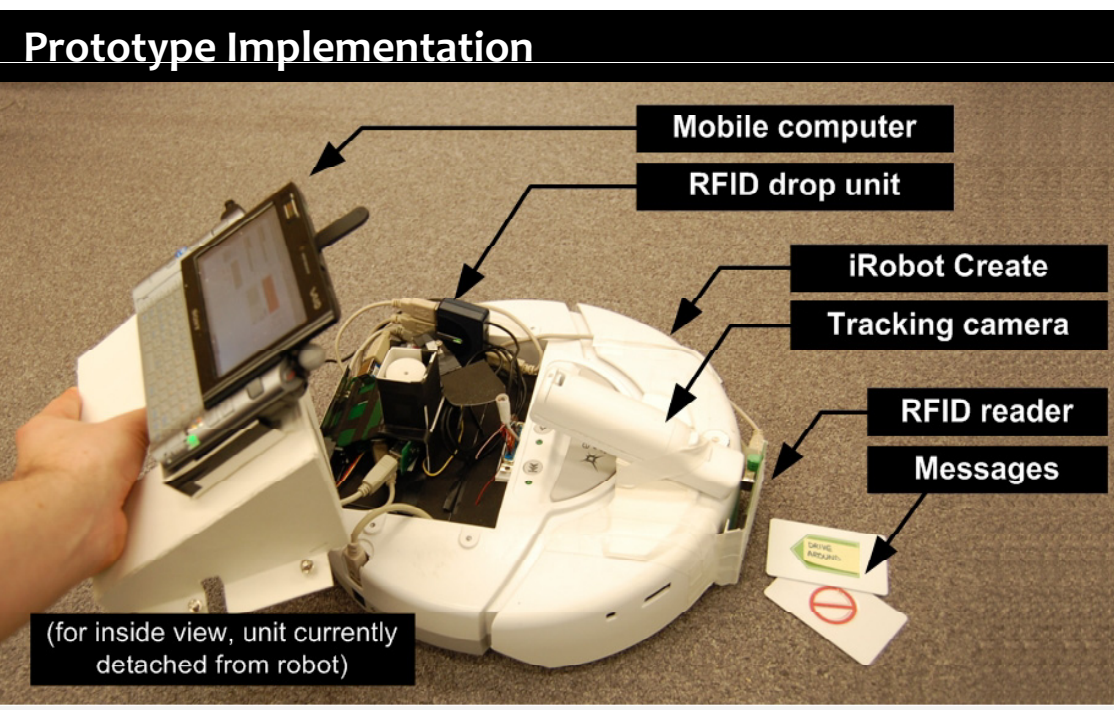

- Robot prototype implementation based on iRobot Create

- Uses RFID message tokens with message content associated to the unique tag identification number

- Active IR for tracking of message tokens at increased distances

- Mechanical unit that enables the robot to place situated messages (by releasing RFID message tokens and associate content to them) 